\title{
Quantitative clinical pharmacology practice for optimal use of antibiotics during the neonatal period
}

ARTICLE in EXPERT OPINION ON DRUG METABOLISM \& TOXICOLOGY · JANUARY 2016

Impact Factor: 2.83 · DOI: 10.1517/17425255.2016.1147559

READS

23

5 AUTHORS, INCLUDING:

Janko Samardzic

University of Belgrade

35 PUBLICATIONS 58 CITATIONS

SEE PROFILE

Marc Pfister

University of Basel

135 PUBLICATIONS 1,737 CITATIONS

SEE PROFILE
Mélanie Wilbaux

University Children's Hospital Basel

14 PUBLICATIONS 33 CITATIONS

SEE PROFILE

Johannes $\mathrm{N}$ van den Anker

Children's National Medical Center

389 PUBLICATIONS 6,994 CITATIONS

SEE PROFILE 


\section{Quantitative clinical pharmacology practice for optimal use of antibiotics during the neonatal period}

\section{Janko Samardzic, Karel Allegaert, Mélanie Wilbaux, Marc Pfister \& John N.} van den Anker

To cite this article: Janko Samardzic, Karel Allegaert, Mélanie Wilbaux, Marc Pfister \& John N. van den Anker (2016): Quantitative clinical pharmacology practice for optimal use of antibiotics during the neonatal period, Expert Opinion on Drug Metabolism \& Toxicology, DOI: 10.1517/17425255.2016.1147559

To link to this article: http://dx.doi.org/10.1517/17425255.2016.1147559

Accepted author version posted online: 27 Jan 2016.

Submit your article to this journal $₫$

Џll Article views: 13

Q View related articles $\longleftarrow$

View Crossmark data $\nearrow$ 
Publisher: Taylor \& Francis

Journal: Expert Opinion on Drug Metabolism \& Toxicology

DOI: $10.1517 / 17425255.2016 .1147559$

\section{REVIEW}

\section{Quantitative clinical pharmacology practice for optimal use of antibiotics during the neonatal period}

Janko Samardzic ${ }^{* 1,2}$, Karel Allegaert ${ }^{3,4}$, Mélanie Wilbaux ${ }^{2}$, Marc Pfister ${ }^{2}$, John N. van den Anker ${ }^{2,5,6}$

${ }^{1}$ Institute of Pharmacology, Clinical Pharmacology and Toxicology, Medical Faculty, University of Belgrade, Serbia

${ }^{2}$ Department of Paediatric Pharmacology, University of Basel Children's Hospital, Basel, Switzerland

${ }^{3}$ Department of Development and Regeneration, KU Leuven, Belgium

${ }^{4}$ Neonatal Intensive Care Unit, University Hospitals Leuven, Belgium

${ }^{5}$ Division of Pediatric Clinical Pharmacology, Children's National Medical Center, Washington, DC, USA

${ }^{6}$ Intensive Care and Department of Pediatric Surgery, Erasmus MC Sophia Children's Hospital, Rotterdam, the Netherlands

\section{*Correspondence:}

Janko Samardzic, MD, PhD

Institute of Pharmacology, Clinical Pharmacology and Toxicology,

Medical Faculty, University of Belgrade,

Dr Subotica 1, 11000 Belgrade, Serbia

Running title: Quantitative clinical pharmacology and antibiotic use in neonates 


\section{Abstract}

Introduction: For safe and effective neonatal antibiotic therapy, knowledge of the pharmacokinetic parameters of antibacterial agents in neonates is a prerequisite. Fast maturational changes during the neonatal period influence pharmacokinetic and pharmacodynamic parameters and their variability. Consequently, the need for applying quantitative clinical pharmacology and determining optimal drug dosing regimens in neonates has become increasingly recognized.

Areas covered: Modern quantitative approaches, such as pharmacometrics, are increasingly utilized to characterize, understand and predict the pharmacokinetics of a drug and its effect, and to quantify the variability in the neonatal population. Individual factors, called covariates in modeling, are integrated in such approaches to explain inter-individual pharmacokinetic variability. Pharmacometrics has been shown to be a relevant tool to evaluate, optimize and individualize drug dosing regimens.

Expert opinion: Challenges for optimal use of antibiotics in neonates can largely be overcome with quantitative clinical pharmacology practice. Clinicians should be aware that there is a next step to support the clinical decision-making based on clinical characteristics and therapeutic drug monitoring, through Bayesian-based modeling and simulation methods. Pharmacometric modeling and simulation approaches permit us to characterize population average, inter-subject and intrasubject variability of pharmacokinetic parameters such as clearance and volume of distribution, and to identify and quantify key factors that influence the pharmacokinetic behavior of antibiotics during the neonatal period.

Keywords: Antibiotics, clinical pharmacology, modeling and simulation, neonate, pharmacometrics, therapeutic drug monitoring 


\section{Article highlights box}

- Low plasma protein binding, changes in body compartments and kidney function influence pharmacokinetic (PK) behavior of antibiotics in neonates.

- Quantitative PK approaches, including pharmacometric modeling and simulation, tailored to neonates are needed to further enhance understanding of PK behavior of antibiotics in neonates.

- Pharmacometric models can integrate the time course of dose-concentration relationships, identify sources of PK variability, and provide a scientific basis for optimizing dosing of antibiotic agents in neonates.

- In conjunction with quantitative pharmacology approaches and appropriate clinical interpretation, therapeutic drug monitoring (TDM) is a valuable tool in neonatal intensive care units.

- Advanced TDM practice leverages Bayesian modeling and simulation methods to ensure safe and effective antibiotic therapy in neonates based on 4 components: (i) measured drug concentrations, (ii) developed population PK models with quantified variability in key PK parameters such as clearance and volume of distribution, (ii) identified time-dependent factors that influence key PK parameters during the first weeks of life, and (iv) characterized PD behavior and target levels of antibiotics in neonates. 


\section{Introduction}

Pharmacokinetics (PK) describes the concentration time profiles of drugs in the body, and includes the processes of absorption, distribution, metabolism and excretion (ADME). The PK profile of drugs in neonates cannot be simply extrapolated from data obtained in older infants, children and adults, since major changes in the physiological characteristics that influence these PK processes happen in early infancy. Furthermore, these developmental changes in ADME do not show the same pattern of maturation $[1,2]$.

Neonates are the group of children from birth up to and including the age of 27 days or the equivalent maturational age ( 44 weeks postmenstrual age) and include both preterm $(<37$ weeks of gestational age) and term neonates [3]. For safe and effective neonatal antibiotic therapy, knowledge of the pharmacokinetic parameters of antibacterial agents in neonates is a prerequisite. Antibiotics are the class of medicines most frequently prescribed in neonates due to the high morbidity and mortality of infectious diseases in preterm and term neonates. Early antibiotic therapy and effective dosing are essential to provide optimal response and limit the spread of resistance [4]. However, fast maturational changes during the neonatal period result in very extensive PK variability, but also affect pharmacodynamics (PD) and may result in differences in drug efficacy, potency and/or toxicity. Across pediatric life, organ size and function change as does body composition, physiology, and cellular function. There is an initial decrease (6-12\%) in birth weight, with a subsequent increase of $50 \%$ in the first 6 weeks of postnatal life. Moreover, weight doubles in the first 3-4 months to result in a 3 fold higher weight at the end of infancy. These physiological trends are further affected by either pharmacotherapy used or pathophysiological processes or co-morbidities [5]. Consequently,

the need for adequate PK/PD studies, prospective validation of quantitative clinical pharmacology approaches in neonates, and determining the correct drug dosing regimen has become increasingly recognized. Quantitative approaches, such as pharmacometrics, can be applied to optimize utilization and fine-tune of antibiotic dosing in neonates. A population approach, based on random 
and limited sampling strategies, has not only improved the feasibility of these studies but has also significantly reduced the burden of classical PK studies.

\subsection{Characteristics of neonatal infections}

Neonatal sepsis is one of the major causes of morbidity and mortality in neonates, especially in low income countries [6]. The risk of sepsis for preterm neonates with a birth-weight between 1000 and $1500 \mathrm{~g}$ in developed countries is $10 \%$, which is around tenfold higher compared to term newborns $[7,8]$. The diagnosis of sepsis is based on both clinical symptoms as well as laboratory findings. Earlyonset infections occur during the first 72 hours of life and are caused by vertical transmission of bacterial pathogens, acquired from the maternal genital tract in utero or during delivery, while lateonset infections, after 3 days of life, include those pathogens acquired from the maternal genital tract as well as after birth through human contact, contaminated equipment or materials, i.e. horizontal transmission [9]. The incidence of documented early-onset neonatal sepsis varies between 0.5 and $1 \%$ of all deliveries, and late-onset sepsis is seen in more than $25 \%$ of very low birth weight infants treated in neonatal intensive care units (NICUs) $[10,11]$. Escherichia coli and Streptococcus Agalactiae are still the two most important causes of early-onset infections, while methicillin resistant coagulase negative staphylococci are the most common organisms involved in the lateonset ones [12].

The high incidence of infections in neonates, the immaturity of the immune system, and the subtle and nonspecific clinical signs and symptoms of infection explain the frequent use of antibiotics in neonates. Due to high morbidity and mortality in this age group, the threshold to initiate antibiotic therapy is low and empirical treatment is usually started while awaiting blood culture results and sensitivity tests. As soon as the results of these tests become available, the initial treatment will be adjusted to a more targeted therapy for that specific pathogen or rapidly stopped when the infection is excluded. 


\subsection{Pharmacokinetic principles of antibiotic use in neonates}

The PK profile of a drug describes its absorption, distribution, metabolism and elimination. This general principle also applies to antibacterial agents, whereby the drug distribution and elimination are considered as main contributors to their PK specific properties in neonates. Absorption refers to the transfer of drugs from the site of administration into the systemic circulation. There are several routes of administration with a different bioavailability, and intravenous administration is recommended for the treatment in neonates to ensure $100 \%$ bioavailability [13]. However, there are additional complicating factors that are specific to intravenous drug administration, such as highly concentrated formulations in the currently available vials. As a consequence, necessary dilution, dosing inaccuracy or errors are more likely to occur in these fragile patients [14]. The more recent introduction of specific pediatric vials showed a reduced variability and improved dosing precision [15]. After absorption, the drug is distributed from the circulation into different bodily fluids, organs and tissues. The distribution of antibiotics in the body is mostly influenced by body composition, but also drug binding to plasma proteins, systemic and regional blood flow and permeability of membranes. Body composition changes with different gestational (GA) and postnatal ages (PNA). Total body water, expressed as a percentage of total body weight, is about $85 \%$ in preterm and $70 \%$ in term neonates, while total body fat in preterm is about $1 \%$ and $15 \%$ in term neonates, respectively. The high proportion of total body water and low proportion of fat tissue results in a lower volume of distribution (Vd) for lipophilic antibiotics like macrolides and fluoroquinolones, and a higher Vd for hydrophilic drugs like aminoglycosides, beta lactams and glycopeptides, as compared to adults. Furthermore, the peak plasma concentration of hydrophilic antibiotics will be delayed and blunted compared to older children or adults [16].

It is well documented that plasma protein binding substantially affects tissue penetration, $\mathrm{Vd}$ and consequently, elimination half-life of antimicrobial agents. First, plasma protein binding reduces the unbound fraction of drug available for bacterial killing, since only the free drug can be distributed throughout the body and has a pharmacological effect. Second, high unbound fractions may result in 
enhanced tissue distribution and an increased Vd of the drug. Finally, it should be taken into account that only the unbound fraction of drug is available for elimination from the vascular compartment [17]. Clinical implications of alterations in drug protein binding are most relevant for drugs which are highly protein bound and have a narrow therapeutic index [18]. The altered drug clearances may lead to significant variations of key PD parameters. Based on in vitro studies, the significant effect of proteins on bacterial killing was confirmed for beta lactams and fluoroquinolones. Zeitlinger et al. demonstrated that albumin substantially impaired the antimicrobial activity of ampicillin, oxacillin and moxifloxacin through a decrease in unbound drug concentration [19]. In neonates, there is reduced concentration of albumin and $\alpha-1$ acid glycoprotein with a decreased drug protein-binding affinity as compared to children and adults. Consequently, the unbound proportion of highly proteinbound drugs will increase. Smits et al. demonstrated that the unbound fraction of cefazolin in neonates was significantly higher than in adults, and it was partly dependent on albuminemia, but also on total drug concentration, indirect bilirubinemia and postmenstrual age [20]. Similar data were also found with flucloxacillin [21]. Therefore, the differences in unbound drug concentration should not solely be attributed to alterations in albumin concentrations, but these also depend on other patients' clinical characteristics. Potentially altered PK of highly protein-bound antibacterial agents may be clinically relevant since they can influence PK parameters and the attainment of PD targets. For antibiotics with high binding affinity for proteins (> 70\%), it is necessary to adjust the dose to maintain the drug concentrations in plasma close to the lower limit of the recommended therapeutic range.

Maturation of drug metabolizing enzymes and kidney function will affect drug elimination in neonates. Rates of hepatic drug metabolism correspond with the low expression of drug metabolizing enzymes at birth, which gradually increases over time [22]. However, drug metabolism is likely only of limited relevance for antibiotics, except when metabolites interfere with therapeutic drug monitoring or metabolites have therapeutic effects [23]. The kidney elimination capacity is also influenced by physiological changes depending on age [24]. The majority of antibiotics used in the 
neonatal population are excreted from the body by the kidneys, and kidney function is the main ratelimiting step for their clearance. Kidney function includes glomerular filtration, tubular secretion and tubular reabsorption, whereby glomerular filtration seems to be more advanced than tubular function at birth. The glomerular filtration rate (GFR) in an individual newborn depends on both maturation until delivery (birth weight) and postnatal maturation (postnatal age). This pattern has been observed using either creatinine clearance estimates [25] or aminoglycoside clearance [26]. In the model proposed by De Cock et al., the developmental changes in clearance of antibiotics (gentamicin, tobramycin and vancomycin) from neonates to adults were considered to be system specific information and were characterized on the basis of one bodyweight-dependent exponent model [27]. Table 1.

Term neonates have a rapid increase in GFR during the first 2 weeks of life and reach adult values by the first year of life, while premature infants show similar trends with a slower initial rise in GFR because nephrogenesis is not completed before 34 weeks of gestation [28,29]. Furthermore, exposure to drugs during the neonatal period might jeopardize the full development of the potential pool of glomeruli and that might have consequences later in life [30,31]. Interestingly, the postnatal maturation results in at least a twofold increase in GFR in the first weeks of life and is due to shifts in intra-renal blood flow [24]. In contrast, renal tubular drug transporters, such as the renal organic anion transporters, are also relevant in the phenotypic clearance of antibiotics (e.g. betalactams) excreted by the kidney [32,33]. The data regarding the maturation and expression of these transporters are currently limited. This necessitates the integration of the ontogeny related knowledge of the elimination route to predict drug specific, PK profiles in neonates. Modern, quantitative approaches, including pharmacometric modeling and model-based antibacterial therapy, provide a powerful research tool to integrate our knowledge into better and safer pharmacotherapy in neonates. Strategic applications of systems pharmacology and pharmacometrics have the great potential to streamline development and optimize utilization of drugs in pediatric patients, including neonates. 


\section{Quantitative clinical pharmacology approaches to optimize use of antibiotics in neonates}

Dynamic maturation and developmental processes during the first weeks of life affect both drug exposure and response and their intra- and inter-subject variability in neonates. Quantitative approaches, such as pharmacometric modeling and simulation, are increasingly utilized to characterize such variability in drug exposure and response in order to individualize and optimize treatment strategies in this vulnerable patient population.

\subsection{Impact of covariates}

A key pharmacokinetic parameter, such as drug clearance, guides drug exposure and as a consequence antibiotic dosing strategies. For this reason, accurate determination of key parameters and their variability in the population of interest, e.g. neonates, is important. Drug clearance can be influenced by demographic, genetic, clinical or any other individual level factor, called covariates in modeling analyses. Pharmacometric covariate models represent mathematical parameterization between such factors and PK parameters and their variability. Based on principles of developmental pharmacology, pharmacometric covariate models should include key components reflecting developmental changes and underlying physiological processes that can affect key parameters such as clearance in neonates: (1) factors of size at birth and growth after birth, (2) factors of agedependent changes and maturational processes that can affect drug transporters and or drug metabolizing enzymes, and (3) factors that affect hepatic and/or kidney function. Body weight is widely used to describe size and growth. Age-dependent maturation can be characterized by various age descriptors, including post-menstrual age (PMA), gestational age (GA), post-natal age (PNA) or post-conceptional age (PCA) $[34,35]$. The incorporation of both GA and PNA in pharmacometric models is recommended to describe two different profiles accounting for ante-natal and post-natal 
maturation processes. As a measure of kidney function serum or plasma creatinine level, creatinine clearance or glomerular filtration rate (GFR) based on measured creatinine, or cystatin C levels are used in the clinical setting $[35,36,37,38]$. Pharmacometric approaches can be utilized to characterize variability in drug exposure and response, and to identify and quantify covariates that influence key PK parameters and their variabilities.

\subsection{Pharmacometric modeling and simulation}

Pharmacometrics is an emerging science of developing and applying mathematical and statistical methods for characterizing, understanding, and predicting a drug's PK, and its effects on biomarker and clinical responses over time $[39,40,41]$. Development of a model aims at translating biological knowledge into mathematical concepts to describe the general trend and variability in the observed data and subsequent predict the pattern in a new, but similar cohort [42].

For many years, PK of antibiotics has been analyzed with classical non-compartmental analysis (NCA), which does not assume any compartments. This simple approach is used to calculate PK parameters such as half-life or area under the serum concentration vs. time curve (AUC), which reflects total drug exposure. AUC is commonly calculated with a trapezoid rule approximating the AUC as a sum of several trapezoids. Such NCA PK analyses are highly dependent on PK sampling schedules and require consistent, rich PK sampling in all study subjects. On the other hand, pharmacometric PK models are built using compartments, representing kinetic concepts that do not necessarily represent any particular region of the body, linked to each other via rate constants. For each compartment, change in drug amounts over time is described by differential equations. Rate constant parameters can be transformed to provide more interpretable PK descriptors, such as drug clearance. There are several advantages of compartmental as compared to NCA analyses: (1) ability to predict drug concentration at any time, (2) flexibility in terms of timing and number of PK samples in study subjects, (3) quantification of covariate effects on key PK parameters, and (4) identification of sources of variability in drug exposure and response. Systems pharmacology models, including physiology-based 
PK models (PBPK), may consist of a large number of compartments representing different organs or tissues in the human body $[43,44]$. Such PBPK models can provide a scientific rationale for first-time dosing in children and facilitate development of drugs in this population. However, further investigations are required and more data on maturational physiology in early infancy need to be collected to expand PBPK models to be used in clinical practice for antibiotics dosing in (pre)term neonates.

Pharmacometric models have usually several components: structural, mathematical component (e.g. differential equations to mass balance between compartments), and statistical components, including those to evaluate and characterize covariate effects on key model parameters. Once structural and covariate components of models have been chosen, parameters and their variability can be estimated, applying the population approach and non-linear mixed-effects modeling [45]. Introduced by Sheiner et al, it is based on a simultaneous analysis of all data from a study population of interest while taking into account that different observations are derived from different individuals. Therefore, such an approach allows for sparse and random sampling designs, which are feasible and preferable in neonatal intensive care units (NICU). These kinds of population PK analyses are robust and permit us to provide a full description of the PK behavior of an antibiotic agent in neonates in terms of estimated average PK parameter, inter-subject and intra-subject variability and key factors that influence PK behavior of antibiotics during the first weeks of life. Quantitative approaches such as pharmacometric modeling and simulation can be used as prior information in computer simulation to describe, explain, and investigate different dosing scenarios. Such approaches have been applied to analyze the PK of renally eliminated antibiotics such as vancomycin, gentamicin, amikacin and meropenem, in order to suggest new dosing recommendations $[46,47,48,49,50]$.

\subsection{Case study: fine-tuning of vancomycin dosing}


Vancomycin is the first choice treatment for serious gram-positive infections, especially involving methicillin-resistant Staphylococcus aureus (MRSA). This narrow spectrum glycopeptide is almost exclusively cleared by glomerular filtration [51]. It is well established that vancomycin dosing in children depends on age, body weight and different maturational aspects. Despite multiple clinical studies and population PK analyses, there is still no consistent and validated dosing recommendation for vancomycin in neonates $[52,53,54,55]$. Capparelli et al. developed a population PK model on a large population, including 374 preterm and term neonates and young infants less than 2 years of age [46]. The authors described organ growth, ante- and post- natal maturation and influence of altered renal function on clearance, by incorporating the combination of weight, GA, PNA and serum creatinine. They validated their model in a set of 67 neonates, and designed a creatinine-based dosing guideline for infants $<60$ days (Table 2). The dosing regimen was based only on serum creatinine, since it can also reflect both growth and maturational changes [56]. They showed a significant improvement, in term of achieving trough levels between $5-10 \mathrm{mcg} / \mathrm{mL}$, compared to published guidelines (Neofax), with a percentage of target attainment from $25 \%$ to $70 \%$. More recent population PK models have been developed, but maturation processes did not include ante-natal maturation (use of PNA alone) or did not differentiate ante- and post- natal maturation (use of PMA) $[47,57,58,59,60]$.

\section{The PK-PD implications and current challenges in antibiotic use in}

\section{neonates}

Pharmacodynamic parameters relate the PK factors to the ability of the antibiotics to kill ('bactericidal') or inhibit the growth ('bacteriostatic') of the infectious agents. The bactericidal antibiotics are overall effective in neonatal infections resulting in different targets and dosing regimens. Thus, aminoglycosides have a concentration-dependent kill characteristic where effect is 
primarily determined by the ratio of antibacterial peak concentration over minimum inhibitory concentration (PEAK/MIC, Fig. 1), while $\beta$-lactam antibiotics have a time-dependent kill characteristic with the time for which the drug serum concentration remains above MIC (T>MIC, Fig. 2) as an optimal predictor of their efficacy [61]. For time-dependent killing antibiotics with moderate persisting killing (e.g. vancomycin), the ratio of the 24-hour area under the concentration (AUC) vS. MIC and time curve is the best predictor for its effectiveness.

While knowledge about the impact of developmental changes on drug PK is increasing, information regarding ontogeny on drug effects (PD) is still limited [2]. Differences in neonatal physiology can affect PD and may result in variability in drug efficacy or toxicity. Age-related developmental changes in the functionality or expression of receptors and differences in disease status may alter the pharmacological response to drugs [62]. However, this variability can only be considered once the PK aspects have been taken into account. Furthermore, extrapolating drug efficacy from adult data to the pediatric population, including neonatal, can streamline drug development only when similar disease progression as well as response to the treatment can be assumed [63]. Immaturity may result in an altered risk of drug toxicity, even a decreased risk. For example, neonates appear to be less susceptible to aminoglycosides renal toxicity compared to children and adults. This PD characteristic may be explained by a reduced intracellular accumulation of these compounds in the tubular epithelial cells of the renal cortex in neonates [64]. More research, especially on PD of antibiotics in the neonatal period and the subsequent clinical implications, is needed. Based on the authors' experience this section addresses the burning challenges faced by clinical pharmacologists, such as the impact of kidney function and relevance of therapeutic drug monitoring, to assure optimal use of antibiotics in neonates.

\subsection{Kidney function and its impact on PK behavior of antibiotics in neonates}

Nephrogenesis starts at week 5-6 of gestation and is completed around week 34-35. The glomerular filtration rate (GFR) in full term neonates is $35 \%$ of adult values, with a rapid increase in GFR during 
the first 2 weeks of life, reaching adult values by the end of the first year of life. Premature infants have similar maturational trends, but with a slower initial GFR rise. Both active tubular secretion and reabsorption are also immature at birth (20-30\% of adult reference values) only to reach adult values in the second part of infancy or beyond $[65,66]$. As a consequence of immature kidney function neonates may develop an accumulation of antibacterial agents that are excreted via the kidneys, with a decrease in plasma clearance and prolonged half-life. Therefore, it is important to have valid biomarkers of kidney function, and to consider an increase of drug dosage interval and/or reduce maintenance dose in this vulnerable group of patients. Clinical characteristics like weight, gestational age, post-menstrual age, co-administration of ibuprofen or perinatal asphyxia hereby perform better when compared to single creatinine measurements [26]. Most neonatal pharmacologists are aware of this and may adjust the dosage of antibiotics accordingly.

However and more recently, some patients - including pediatric patients - have been documented to display 'paradoxically' increased clearance of antibacterial agents, so-called glomerular hyperfiltration related to their disease state $[67,68]$. Unfortunately, this aspect has not yet been sufficiently explored in early infancy. In general, glomerular hyperfiltration means that the renal clearance of antibiotics is beyond the anticipated physiological range. Accurately defining this is difficult, and one of the most sensible definitions is a GFR $>10 \%$ higher than the normal limits [67]. Glomerular hyperfiltration has been documented for beta lactams in adult patients with sepsis, resulting in slower eradication of the infection or even introducing resistance $[69,70,71]$. The pathophysiological mechanism responsible for glomerular hyperfiltration in sepsis is not entirely clear, but could involve altered renal blood flow. In animal studies, renal blood flow has been shown to increase in parallel with cardiac output in early sepsis, while it is diminished in a later phase of sepsis, resulting in decreased creatinine clearance [72]. These data confirm that in critically ill patients renal clearance might be increased in the acute phase, with significant clinical implications at the start of antibacterial treatment. To the best of our knowledge, there are no data on glomerular hyperfiltration in the critically ill neonates, but we might certainly expect rapid renal changes during 
neonatal infections. Since the presence of a weak correlation between the dose administered and the concentration reached is one of the circumstances to consider TDM, the variability in renal clearance is an argument in support of TDM in neonates.

\subsection{Therapeutic drug monitoring of antibiotics in neonates: wasted blood or justified practice?}

Current data suggest that the greatest benefit of TDM can be expected within targeted or special subpopulations: those at the extremes of age, those with severely impaired kidney function, and those using specific drugs, such as immunosuppressive, antineoplastic or anticonvulsant agents. Consequently, neonates fit into this, but experiencing some specific issues of neonatal samples, such as low volume, plasma protein content or hyperbilirubinemia. In addition, other issues - including non PK - make accurate dosing and monitoring more difficult in neonates. There is often not an appreciation of the substantial variability in the rate of drug delivery from the intravenous line in neonates, resulting in difficulties in accurately determining the drug PK-PD relationship [14]. On the other hand, when the appropriateness of TDM for a particular drug is considered, anticipated quantitative relationship between concentration and effects, a narrow therapeutic index and/or significant consequences of therapeutic failure are all in favor of using TDM. Furthermore, routine TDM is only rational when there is a sufficiently precise and accurate analytical technique, as well as well knowledge concerning the PK of the drug and relevant covariates in the specific patient population (e.g. weight, age, comorbidity).

Neonates, with rapid maturational changes, benefit from TDM for commonly used drugs, but mostly for aminoglycosides and glycopeptides $[59,73]$. The inconsistency of dosing guidelines for achieving therapeutic serum concentrations using empiric dosing regimens underlines the importance of appropriate TDM in neonates. Large inter-individual differences in PK parameters secondary to gestational age, co-medication and predictive performance of initial empiric therapy might be improved by TDM. However, even when it is implemented, any measurement needs to be integrated into the clinical setting of the individual patient. Thus, some relevant issues related to the validated 
target range and time of sampling may occur. For many drugs, target concentrations are not clearly defined in children, but only based on data provided in adults. The antibacterial target range validated in adults is used in children, including neonates, although additional data are needed to validate that these bacteriological targets are associated with efficacy in neonatal infections. TDM sampling for aminoglycosides is traditionally performed at steady state, around the fourth dose. For neonates, this may be impractical, since neonatal antimicrobial therapy is often empiric and may be discontinued after 72 hours in case of negative blood cultures. Early sampling with subsequent Bayesian approaches may be a possible alternative; however this needs to be further evaluated. Kadambari et al. demonstrated considerable variation amongst gentamicin monitoring protocols in different neonatal units in the UK, with no clear justification [74]. A dosing interval of 24 to 48 hours is usually recommended because of reduced clearance and prolonged half-life in neonates. The similar conclusion was reached for vancomycin, where the samples were also taken at different points during treatment. Most NICUs take samples around the third dose. The peak serum vancomycin concentrations have not been consistently associated with efficacy or toxicity in neonates, therefore routine sampling of peak serum concentrations may not be necessary. Instead, a shift towards taking only routine trough serum concentrations provides optimal efficacy regarding TDM in neonates receiving vancomycin [75]. Furthermore, the bioanalytical assays validated to measure antibacterial concentrations in neonates may differ. Recently, in a single NICU center we have confirmed the relevance of vancomycin bio-assays, and particularly target values may be substantially different between assays [76,77]. Such studies strongly suggest that dosage individualization and monitoring should not only consider clinical characteristics, but should also be tailored to the method of drug quantification. In conjunction with quantitative pharmacology approaches and appropriate clinical interpretation, TDM is a valuable tool in NICU.

\section{Conclusion}


Due to highly dynamic period of neonatal development, many covariates such as clinical characteristics influence PK in neonates, resulting in large between- and within-patient variability both in drug disposition and effects. A quantitative approach, such as pharmacometric modeling and simulation, has been shown to be a relevant tool to evaluate and optimize drug dosing regimens, as covariates, explaining inter-individual PK variability, may be integrated in a new dosing recommendation. Based on this strategy, PK knowledge and dosing recommendations in neonates have been significantly improved over the last decades.

Models that can integrate the time courses of dose-concentration relationships and quantification of variability provide a scientific basis for optimizing antibiotics dosing in neonates. However, they are not routinely used by practicing neonatologists and pediatricians. For this reason simple bed side decision tools are developed to support clinicians in individualizing and optimizing dosing of antibiotics in neonates, such as the EzeCHiel platform [http://www.ezechiel.ch/].

\section{Expert Opinion}

Antibiotics are the class of medicines most frequently prescribed in neonates due to the high morbidity and mortality of infectious diseases in preterm and term neonates. Early antibiotic therapy and effective dosing are essential to provide optimal response and limit the spread of resistance [4]. Applied pharmacokinetics is an opportunity as well as a challenge for optimal use of antibiotics in neonates. In Figure 3, we demonstrate the progress of quantitative clinical pharmacology practice over time: from simple and very often inaccurate dosing regimens, through complex dosing and model-based antibacterial therapy, to integrated and balanced PK/PD knowledge with an individualized approach $[76,78,79,80,81]$. 
Pharmacokinetics (PK) of antibiotics has been analyzed for years with classical non-compartmental analyses. This simple approach is used to calculate PK parameters such as half-life or area under the serum concentration vs. time curve, which reflects total drug exposure. However, such noncompartmental PK analyses are highly dependent on PK sampling schedules and require consistent PK sampling in all study subjects. In contrast, pharmacometric models are built using compartments, representing kinetic concepts that do not necessarily represent any particular region of the body, linked to each other via rate constants. Individual factors, called covariates in modeling, are integrated in such approaches to explain inter-individual pharmacokinetic variability. Quantitative clinical pharmacology approaches, such as pharmacometric modeling and simulation, permit us in the future to provide a full description of the PK behavior of antibiotic agents in neonates in terms of population average PK parameter, inter-subject and intra-subject variability, and key factors that influence PK behavior of antibiotics during the neonatal period. Such pharmacometric approaches have been already applied to characterize PK behavior of renally eliminated antibiotics, such as vancomycin, gentamicin, amikacin and meropenem, in order to suggest new dosing recommendations $[46,47,48,49,50]$. Furthermore, characterization of relationships between PK and PD of antibiotics in neonates is essential to optimize treatment strategies in neonates. Ramos-Marti et al. used pharmacometric approaches to investigate optimal dosing of vancomycin in neonates by bridging data from dynamics in vitro and in vivo experiments to neonates [82].

To further individualize and fine-tune dosing strategies of antibiotics in neonates, especially compounds with a relatively narrow therapeutic index, TDM approaches are encouraged. Since the presence of a weak correlation between the dose administered and the concentration reached is one of the circumstances to consider TDM, whereas the variability in renal clearance is another argument in support of TDM in neonates. Clinicians should be aware that there is a next step to support the clinical decision making based on the clinical characteristics and the TDM measurement. This next step is through advanced TDM practice that leverages Bayesian modeling and simulation methods to 
guide dosing of antibiotics in neonates, based on 4 components: (i) measured drug concentrations, (ii) developed population PK models with quantified variability in key PK parameters such as clearance and volume of distribution, (ii) identified time-dependent factors that influence key PK parameters during the first weeks of life, and (iv) characterized PD behavior and target levels of antibiotics in neonates. With such quantitative clinical pharmacology approaches dose recommendations can be optimized and individualized, ensuring safe and effective antibiotic therapy in neonates.

\section{Declaration of Interest}

J Samardzic is supported by ERAWEB II scholarship for postdoctoral program at the KU Leuven, Belgium (2014-2015) and Ministry of Education, Science and Technological Development of the Republic of Serbia (project No. 175076). K Allegaert is supported by the Fund for Scientific Research, Flanders (fundamental clinical investigatorship 1800214N) and the research activities are further facilitated by the agency for innovation by Science and Technology in Flanders (IWT) through the SAFEPEDRUG project (IWT8SBO 130033). JN van den Anker is supported by NIH (K24DA027992, R01HD048689, U54HF071601) and the European Commission (TINM [223614], TINN2 [260908], NEUROSIS [223060]). The authors have no other relevant affiliations or financial involvement with any organization or entity with a financial interest in or financial conflict with the subject matter or materials discussed in the manuscript apart from those disclosed. 
Figure 1

Aminoglycosides: PEAK/MIC - optimal predictor of their efficacy

PEAK - antibacterial peak concentration

MIC - minimum inhibitory concentration

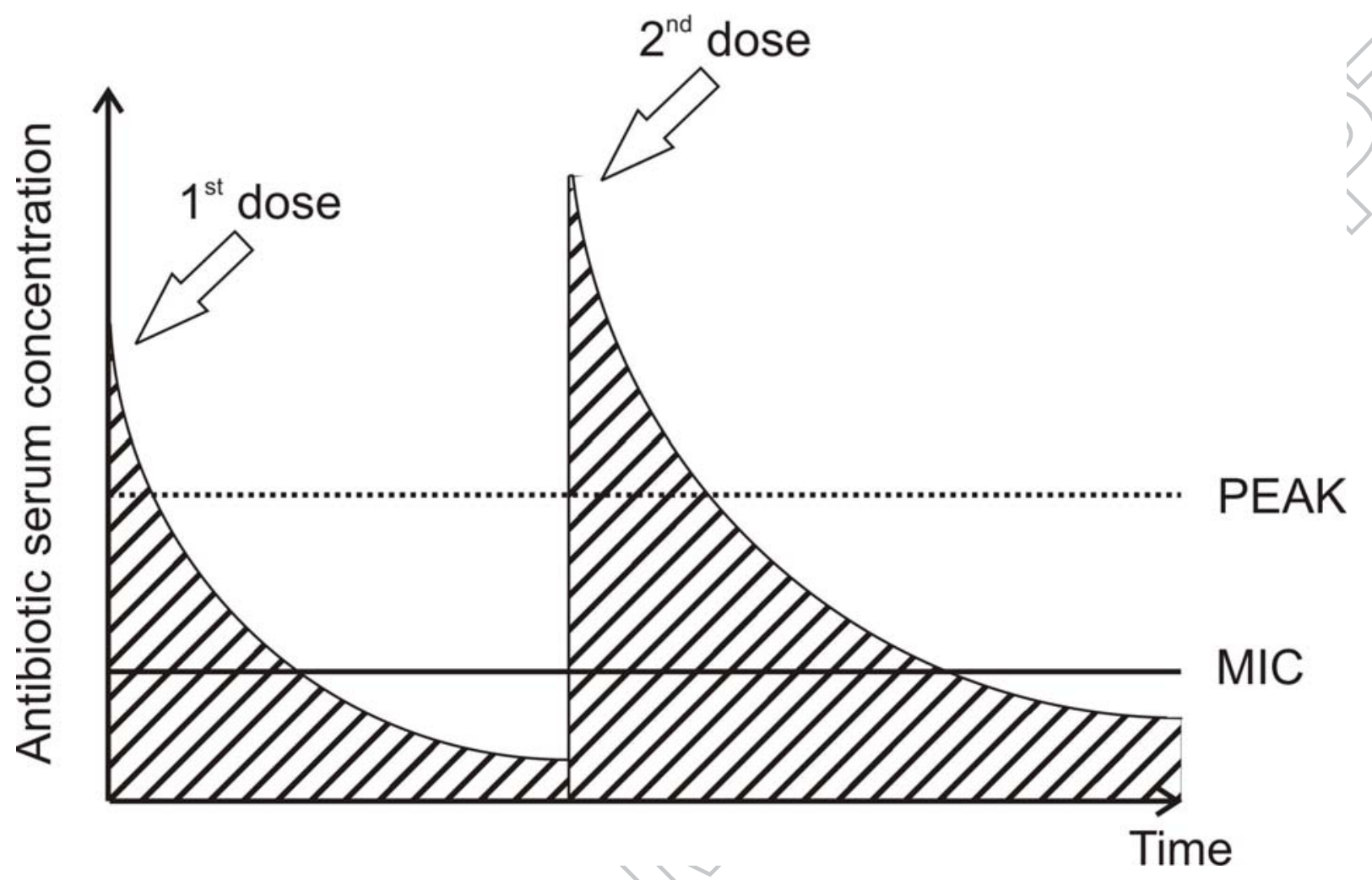




\section{Figure 2}

Penicillines: Time above MIC - optimal predictor of their efficacy MIC - minimum inhibitory concentration

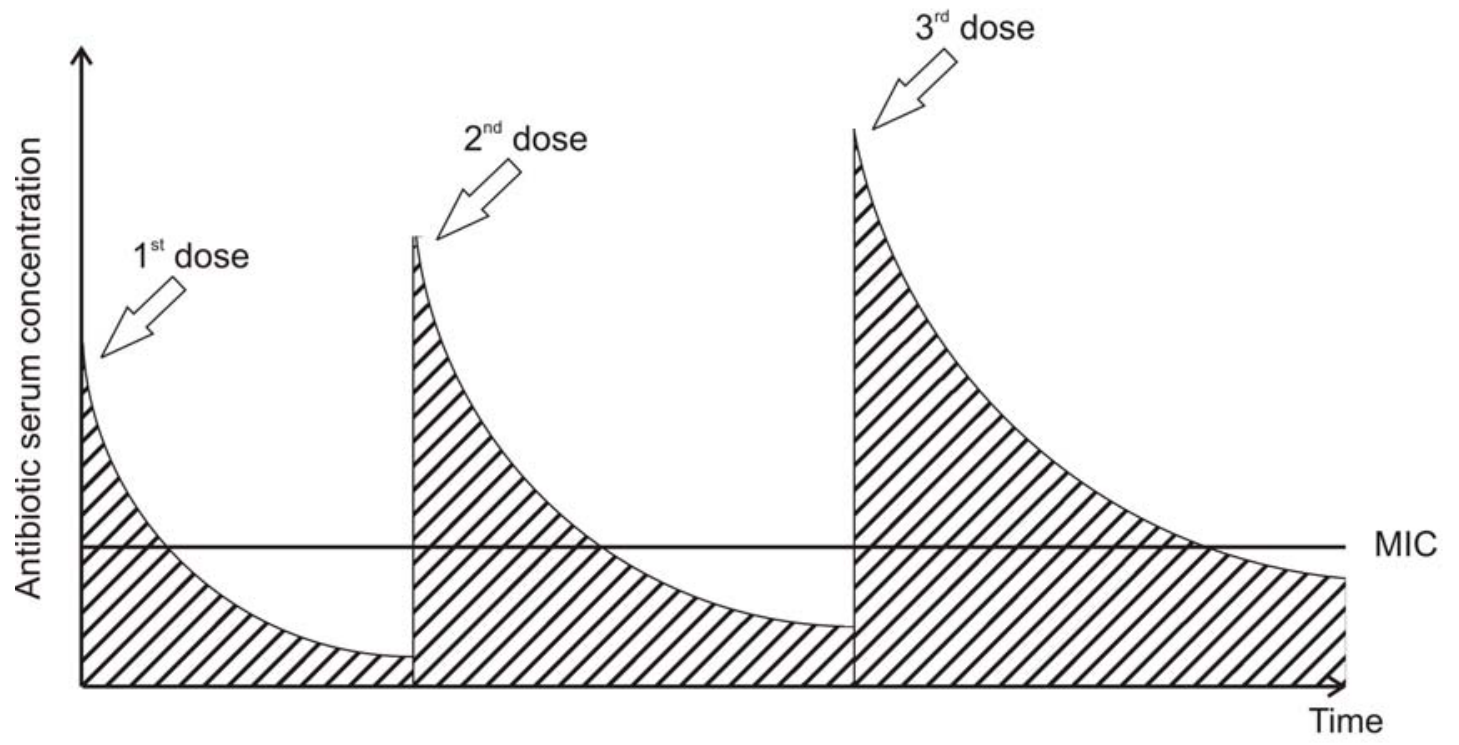




\section{Figure 3}

Progress of quantitative clinical pharmacology practice for safe and effective antibiotic therapy in neonates

TDM - therapeutic drug monitoring

PK/PD - pharmacokinetics/pharmacodynamics

\section{Former practice}

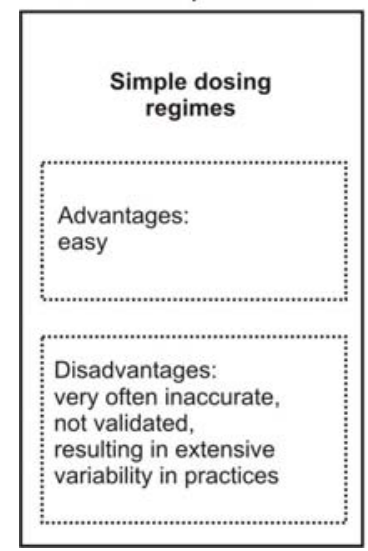

Current practice

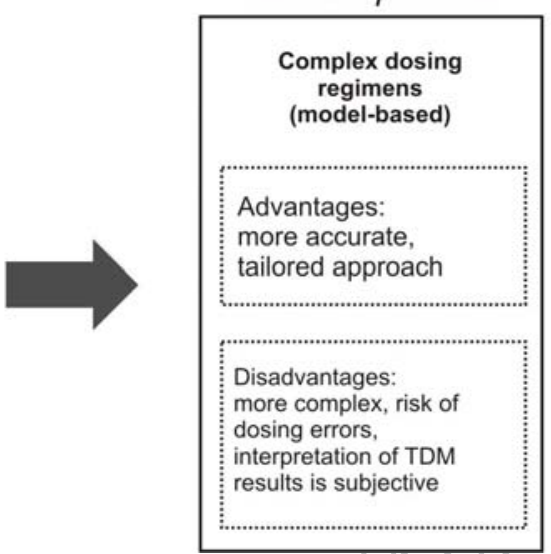

Future practice

Complex dosing regimens with Bayesian-based modeling and simulation

Advantages:

integrated knowledge

on PK/PD,

individualized approach

Disadvantages:

sophisticated approach,

need for multidisciplinary

collaboration and accurate

and valid TDM and

measurement techniques 


\section{ibliography}

Papers of special note have been highlighted as:

* of interest

** of considerable interest

1. Van den Anker JN, Schwab M, Kearns GL. Developmental Pharmacokinetics. Handb Exp Pharmacol 2011;205:51-75

2. Samardzic J, Allegaert K, Bajcetic M. Developmental pharmacology: A moving target. Int I Pharm 2015;492:335-7

3. Williams K, Thomson D, Seto I, et al. Standard 6: age groups for pediatric trials. Pediatrics 2012;129:S153-60

4. Leekha S, Terrell CL, Edson RS. General principles of antimicrobial therapy. Mayo Clinic proceedings. 2011;86:156-67

5. Yaffe SJ, Aranda JV. Neonatal and pediatric pharmacology: therapeutic principles in practice. 4th edn, Philadelphia: Lippincott Williams Wilkins, 2011

6. Black RE, Cousens S, Johnson $\mathrm{HL}$, et al. Global, regional, and national causes of child mortality in 2008: a systematic analysis. The Lancet 2010;375:1969-87

7. Kaufman D, Fairchild KD. Clinical microbiology ofbacterial and fungal sepsis in very-low-birthweight infants. Clin Microbiol Rev 2004; 17:638-80

8. Ohlin A, Björkman L, Serenius F, et al. Sepsis as a risk factor for neonatal morbidity in extremely preterm infants. Acta Paediatr 2015, DOI 10.1111/apa.13104

9. Remington JS, Klein JO, Wilson CB, et al. Infectious Diseases of the Fetus and Newborn Infant. 7th ed. Philadelphia, PA: Elsevier Saunders, 2011 
10. Stoll BJ, Gordon T, Korones SB, et al. Late-onset sepsis in very low birth weight neonates: a report from the national institute of child health and human development neonatal research network. J Pediatr 1996;129:63-71

11. Stoll BJ, Hansen NI, Sanchez PJ, et al. Eunice Kennedy Shriver National Institute of Child Health and Human Development Neonatal Research Network. Early onset sepsis: the burden of group B streptococcal and E. coli disease continues. Pediatrics 2011;127:817-26

12. van den Anker JN. How to optimize the evaluation and use of antibiotics in neonates. Early Hum Dev 2014;90:S10-2

\section{** Key paper on the need for more rational approach in the neonatal use of antibiotics}

13. MacDonald MG, Mullett MD, Seshia MMK. Avery's Neonatology: Pathophysiology \& Management of the Newborn. 6th ed. Philadelphia, PA: Lippincott Williams \& Wilkins, 2005

14. Sherwin CM, Medlicott NJ, Reith DM, Broadbent RS. Intravenous drug delivery in neonates: lessons learnt. Arch Dis Child 2014;99:590-4

15. Allegaert K, Anderson BJ, Vrancken M, et al. Impact of a paediatric vial on the magnitude of systematic medication errors in neonates. Paediatr Perinat Drug Ther 2006; 7:59-63

16. Samardzic J, Turner MA, Bax R, Allegaert K. Neonatal medicines research: challenges and opportunities. Expert Opin Drug Metab Toxicol 2015;11:1041-52

17. Ulldemolins $M$, Roberts $J A$, Rello J, et al. The effects of hypoalbuminaemia on optimizing antibacterial dosing in critically ill patients. Clin Pharmacokinet 2011;50:99-110

18. Roberts JA, Pea F, Lipman J. The clinical relevance of plasma protein binding changes. Clin Pharmacokinet 2013;52:1-8

* extensive review on the clinical relevance of plasma protein binding 
19. Zeitlinger MA, Sauermann R, Traunmüller $F$, et al. Impact of plasma protein binding on antimicrobial activity using time-killing curves. J Antimicrob Chemother 2004;54:876-80

20. Smits A, Kulo A, Verbesselt R, et al. Cefazolin plasma protein binding and its covariates in neonates. Eur J Clin Microbiol Infect Dis 2012;31:3359-65

21. Pullen J, Stolk LM, Degraeuwe PL, et al. Protein binding of flucloxacillin in neonates. Ther Drug Monit 2007;29:279-83

22. Hines RN. Developmental expression of drug metabolizing enzymes: impact on disposition in neonates and young children. Int J Pharm 2013;452:3-7

23. Ahsman MJ, Wildschut ED, Tibboel D, Mathot RA. Pharmacokinetics of cefotaxime and desacetylcefotaxime in infants during extracorporeal membrane oxygenation. Antimicrob Agents Chemother 2010;54:1734-41

24. Schreuder MF, Bueters RR, Allegaert K. The interplay between drugs and the kidney in premature neonates. Pediatr Nephrol 2014;29:2083-91

25. Vieux R, Hascoet JM, Merdariu D, et al. Glomerular filtration rate reference values in very preterm infants. Pediatrics 2010;125:e1186-92

26. De Cock RF, Allegaert K, Sherwin CM, et al. A neonatal amikacin covariate model can be used to predict ontogeny of other drugs eliminated through glomerular filtration in neonates. Pharm Res $2014,31: 754-67$

27. De Cock RF, Allegaert K, Brussee JM, et al. Simultaneous pharmacokinetic modeling of gentamicin, tobramycin and vancomycin clearance from neonates to adults: towards a semiphysiological function for maturation in glomerular filtration. Pharm Res 2014;31:2643-54

28. Rhodin MM, Anderson BJ, Peters AM, et al. Human renal function maturation: a quantitative description using weight and postmenstrual age. Pediatr Nephrol 2009;24:67-76 
29. De Cock RF, Allegaert K, Schreuder MF, et al. Maturation of the glomerular filtration rate in neonates, as reflected by amikacin clearance. Clin Pharmacokinet 2012;51:105-17

30. Bueters RR, Kusters LJ, Klaasen A, et al. Antibiotics and renal branching morphogenesis: comparison of toxicities. Pediatr Res 2014;76:508-14

31. Girardi A, Raschi E, Galletti S, et al. Drug-induced renal damage in preterm neonates: state of the art and methods for early detection. Drug Saf 2015;38:535-51

32. Yaffe SJ, Aranda JV, Anker van den JN. Neonatal and pediatric pharmacology: therapeutic principles in practice. $3^{\text {rd }}$ edn, Philadelphia: Lippincott Williams Wilkins, 2005

33. Chen N, Aleksa K, Woodland C, et al. Ontogeny of drug elimination by the human kidney. Pediatr Nephrol 2006;21:160-8

34. Engle WA, American Academy of Pediatrics Committee on Fetus and Newborn. Age terminology during the perinatal period. Pediatrics 2004,114:1362-4

35. Rodieux F, Wilbaux M, van den Anker NN, Pfister M. Effect of Kidney Function on Drug Kinetics and Dosing in Neonates, Infants, and Children. Clin Pharmacokinet 2015; [Epub ahead of print]

36. Schwartz GJ, Haycock GB, Edelmann CM Jr, Spitzer A. A simple estimate of glomerular filtration rate in children derived from body length and plasma creatinine. Pediatrics 1976;58:259-63

37. Kandasamy $Y$, Smith R, Wright IM. Measuring cystatin $C$ to determine renal function in neonates. Pediatric critical care medicine: a journal of the Society of Critical Care Medicine and the World Federation of Pediatric Intensive and Critical Care Societies 2013;14:318-22

38. Brou NA, Jacqz-Aigrain E, Zhao W. Cystatin C as a potential biomarker for dosing of renally excreted drugs. Br J Clin Pharmacol 2015;80:20-7 
39. Pfister M, D'Argenio DZ. The emerging scientific discipline of pharmacometrics. J Clin Pharmacol 2010;50:6S

40. De Cock RF, Piana C, Krekels EH, et al. The role of population PK-PD modelling in paediatric clinical research. Eur J Clin Pharmacol 2011;67:5-16

\section{** review on the role of modelling and simulation in paediatric pharmacology}

41. van der Graaf PH. CPT: Pharmacometrics and Systems Pharmacology. CPT: pharmacometrics \& systems pharmacology 2012;1:e8

42. Mould DR, Upton RN. Basic concepts in population modeling, simulation, and model-based drug development-part 2: introduction to pharmacokinetic modeling methods. CPT: pharmacometrics \& systems pharmacology 2013;2:e38

43. Johnson TN, Rostami-Hodjegan A. Resurgence in the use of physiologically based pharmacokinetic models in pediatric clinical pharmacology: parallel shift in incorporating the knowledge of biological elements and increased applicability to drug development and clinical practice. Paediatr Anaesth 2011;21:291-301

44. Jones $\mathrm{H}$, Rowland-Yeo K. Basic concepts in physiologically based pharmacokinetic modeling in drug discovery and development. CPT: pharmacometrics \& systems pharmacology 2013;2:e63

45. Sheiner LB. The population approach to pharmacokinetic data analysis: rationale and standard data analysis methods. Drug metabolism reviews 1984;15:153-71

46. Capparelli EV, Lane JR, Romanowski GL, et al. The influences of renal function and maturation on vancomycin elimination in newborns and infants. J Clin Pharmacol 2001;41:927-34

\section{** extensive vancomycin population PK study with proposed dosing regimen}


47. Allegaert K, Anderson BJ, van den Anker JN, et al. Renal drug clearance in preterm neonates: relation to prenatal growth. Ther Drug Monit 2007;29:284-91

48. Smith PB, Cohen-Wolkowiez M, Castro LM, et al. Population pharmacokinetics of meropenem in plasma and cerebrospinal fluid of infants with suspected or complicated intra-abdominal infections. Pediatr Infect Dis J 2011;30:844-9

49. Van den Anker JN, Pokorna P, Kinzig-Schippers $M$, et al. Meropenem pharmacokinetics in the newborn. Antimicrob Agents Chemother 2009;53:3871-9

50. Fuchs A, Guidi M, Giannoni E, et al. Population pharmacokinetic study of gentamicin in a large cohort of premature and term neonates. Br J Clin Pharmacol 2014;78:1090-101

51. Golper TA, Noonan HM, Elzinga L, et al. Vancomycin pharmacokinetics, renal handling, and nonrenal clearances in normal human subjects. Clin Pharmacol Ther 1988;43:565-70

52. Liu C, Bayer A, Cosgrove SE, et al. Clinical practice guidelines by the infectious diseases society of america for the treatment of methicillin-resistant Staphylococcus aureus infections in adults and children. Clin Infect Dis 2011;52:e18-55

53. Jacqz-Aigrain $E$, Zhao W, Sharland M, van den Anker JN. Use of antibacterial agents in the neonate: 50 years of experience with vancomycin administration. Semin Fetal Neonatal Med $2013 ; 18: 28-34$

54. Vandendriessche A, Allegaert K, Cossey V, et al. Prospective validation of neonatal vancomycin dosing regimens is urgently needed. Curr Ther Res Clin Exp 2014;76:51-7

55. Jacqz-Aigrain $\mathrm{E}$, Leroux $\mathrm{S}$, Zhao $\mathrm{W}$, et al. How to use vancomycin optimally in neonates: remaining questions. Expert Rev Clin Pharmacol 2015;8:635-48 
56. Bhongsatiern JJ, Stockmann C, Yu T, et al. Renal function descriptors in neonates: Which creatinine-based formula best describes vancomycin clearance? J Clin Pharmacol 2015, DOI 10.1002/jcph.650

57. Kimura T, Sunakawa K, Matsuura N, et al. Population pharmacokinetics of arbekacin, vancomycin, and panipenem in neonates. Antimicrob Agents Chemother 2004;48:1159-67

58. Mehrotra N, Tang L, Phelps SJ, Meibohm B. Evaluation of vancomycin dosing regimens in preterm and term neonates using Monte Carlo simulations. Pharmacotherapy 2012;32:408-19

59. Zhao W, Lopez E, Biran V, et al. Vancomycin continuous infusion in neonates: dosing optimisation and therapeutic drug monitoring. Arch Dis Child 2013;98:449-53

60. Frymoyer A, Hersh AL, El-Komy MH, et al. Association between vancomycin trough concentration and area under the concentration-time curve in neonates. Antimicrob Agents Chemother 2014;58:6454-61

61. Roberts JA, Lipman J. Antibacterial dosing in intensive care: pharmacokinetics, degree of disease and pharmacodynamics of sepsis. Clin Pharmacokinet 2006;45:755-73

62. Mulla H. Understanding developmental pharmacodynamics: importance for drug development and clinical practice. Paediatr Drugs 2010;12:223-33

63. Dunne J, Rodriguez WJ, Murphy MD, et al. Extrapolation of adult data and other data in pediatric drug-development programs. Pediatrics 2011;128:e1242-9

64. Kent A, Turner MA, Sharland M, et al. Aminoglycoside toxicity in neonates: something to worry about ? Expert Rev Anti Infect Ther 2014;12:319-31

65. Standing JF, Tuleu C. Paediatric formulations--Getting to the heart of the problem. Int J Pharm 2005;300:56-66 
66. de Wildt SN. Profound changes in drug metabolism enzymes and possible effects on drug therapy in neonates and children. Expert Opin Drug Metab Toxicol 2011;7:935-48

67. de Lange DW. Glomerular hyperfiltration of antibiotics. Neth J Crit Care 2013;17:10-4

\section{* glomerular hyperfiltration of antibiotics as an issue}

68. de Cock P, Standing J, Barker C, et al. Augmented renal clearance implies a need for increased amoxicillin-clavulanate dosing in critically ill children. Arch Dis Child 2016;101:e1

69. Chytra I, Stepan M, Benes J, et al. Clinical and microbiological efficacy of continuous versus intermittent application of meropenem in critically ill patients: a randomized open-label controlled trial. Crit Care 2012;16:R113

70. Tröger U, Drust A, Martens-Lobenhoffer J, et al. Decreased meropenem levels in Intensive Care Unit patients with augmented renal clearance: benefit of therapeutic drug monitoring. Int J Antimicrob Agents 2012;40:370-2

71. Udy AA, Varghese JM, Altukroni $M$, et al. Subtherapeutic initial $\beta$-lactam concentrations in select critically ill patients: association between augmented renal clearance and low trough drug concentrations. Chest 2012;142:30-9

72. Prowle JR, Molan MP, Hornsey E, Bellomo R. Measurement of renal blood flow by phase-contrast magnetic resonance imaging during septic acute kidney injury: a pilot investigation. Crit Care Med 2012;40:1768-76

73. van den Anker JN, Allegaert K. Pharmacokinetics of aminoglycosides in the newborn. Curr Pharm Des 2012;18:3114-8

74. Kadambari S, Heath PT, Sharland M, et al. Variation in gentamicin and vancomycin dosage and monitoring in UK neonatal units. J Antimicrob Chemother 2011;66:2647-50

* variation in gentamicin and vancomycin dosing and TDM guidance across UK NICUs 
75. de Hoog M, Mouton JW, van den Anker JN. Vancomycin: pharmacokinetics and administration regimens in neonates. Clin Pharmacokinet 2004;43:417-40

76. Zhao W, Kaguelidou F, Biran V, et al. External Evaluation of Population Pharmacokinetic Models of Vancomycin in Neonates: The transferability of published models to different clinical settings. $\mathrm{Br} J$ Clin Pharmacol 2013;75:1068-80

77. Samardzic J, Smits A, Cossey V, et al. To what extent does the vancomycin assay contribute to the variability in vancomycin concentration estimates in neonates? Arch Dis Child 2016;101:e1

78. Allegaert K, van den Anker JN. Creatinine-based vancomycin dosing regimens in neonates: there is more to consider than the variation in drug assay. Pharmacotherapy 2012;32:e174;discussion e175

79. Allegaert K, van den Anker JN. Adverse drug reactions in neonates and infants: a populationtailored approach is needed. Br J Clin Pharmacol 2015;80:788-95

80. Metsvaht $T$, Nellis $G$, Varendi $H$, et al. High variability in the dosing of commonly used antibiotics revealed by a Europe-wide point prevalence study: implications for research and dissemination. BMC Pediatr 2015;15:41

** recent analyses on the variability in the dosing of frequently used antibiotics in European NICUs

81. Smits A, De Cock RF, Allegaert K, et al. Prospective evaluation of a model-based dosing regimen for amikacin in preterm and term neonates in clinical practice. Antimicrob Agents Chemother 2015; 59:6344-51

82. Ramos-Martín V, Johnson A, Livermore J, et al. Pharmacodynamics of vancomycin for CoNS infection: experimental basis for optimal use of vancomycin in neonates. J Antimicrob Chemother 2016, DOI 10.1093/jac/dkv451 
Table 1.

Clearance of gentamicin, tobramycin and vancomycin from neonates to adults, based on bodyweight-dependent exponent model [27]

\begin{tabular}{|l|l|l|l|}
\hline & Gentamicin L/h (L/kg/h) & Tobramycin L/h (L/kg/h) & Vancomycin L/h (L/kg/h) \\
\hline Neonate (4 kg) & $0.21(0.05)$ & $0.28(0.07)$ & $0.39(0.097)$ \\
\hline Infant (10 kg) & $0.65(0.065)$ & $0.90(0.09)$ & $1.55(0.155)$ \\
\hline Child (20 kg) & $1.38(0.069)$ & $1.84(0.092)$ & $2.56(0.128)$ \\
\hline Adult (60 kg) & $4.00(0.067)$ & $5.34(0.089)$ & $(0.43(0.124)$ \\
\hline
\end{tabular}

Table 2.

Vancomycin dosing regimen proposed by Capparelli et al. [46]

\begin{tabular}{|l|l|l|}
\hline Creatinine (mg/dL) & Dose (mg/kg) & Dosing interval (h) \\
\hline$\geq 1.7$ & 15 & 48 \\
\hline $\mathbf{1 . 3}-\mathbf{1 . 6}$ & 10 & 24 \\
\hline $\mathbf{1 . 0}-\mathbf{1 . 2}$ & 15 & 24 \\
\hline $\mathbf{0 . 7}-\mathbf{0 . 9}$ & 20 & 24 \\
\hline$\leq \mathbf{0 . 6}$ & 15 & 12 \\
\hline
\end{tabular}

